\title{
Optimization, design, and modeling of ferrite core geometry for inductive wireless power transfer
}

\author{
Ludvik Strauch ${ }^{\mathrm{a}, *}$, Mojca Pavlin ${ }^{\mathrm{b}}$ and Vladimir Boštjan Bregar ${ }^{\mathrm{a}, \mathrm{b}}$ \\ ${ }^{a}$ Kolektor Group, Kolektor Sikom, Magma, Ljubljana, Slovenia \\ ${ }^{\mathrm{b}}$ Faculty of Electrical Engineering, University of Ljubljana, Ljubljana, Slovenia
}

\begin{abstract}
This paper presents a new design of ferrite core made of ferrite bars positioned in radial geometry. New core design is used in inductive wireless power transfer where the optimal design of ferrite bars was analyzed. Numerical simulations of seven ferrite bars geometries were performed in 3D models using Comsol Multiphysics in terms of the coupling coefficient. Two optimal designs of three and nine ferrite bars geometries were used for parameterization of geometric parameters. Both simulations of three and nine ferrite bars geometries were verified by measurements. Optimal design of nine ferrite bars geometry is proposed for use in wireless power transfer because of low consumption of ferrite material.

Ferrite bars geometry was also used as a magnetic shield to reduce magnetic fields which may interfere with the electronics nearby. Magnetic flux density in ferrite bars is low enough that prevents ferrite bars from the saturation. The angular alignment of optimal design of nine ferrite bars on the transmitter and receiver side was negligible as the difference in the coupling coefficient was $0.13 \%$. Advantages of using optimal design of nine ferrite bars geometry are: high value of the coupling coefficient, low consumption of ferrite material, and reduced weight.
\end{abstract}

Keywords: Ferrite cores, optimization, finite element method, wireless power transfer

\section{Introduction}

The inductive wireless power transfer is today one of the most interested technologies, as it can be used in numerous applications. It can be used for powering electric vehicles [1-7], monorail systems [8- 10], mobile phones [11|12], and other low power mobile devices [13-15]. In general, inductive system [2, 12] uses a transmitter and receiver coil where ferrite cores are often used to improve wireless power transfer [3[16]. When designing an inductive system, two important parameters, the coupling coefficient and value of magnetic flux density in terms of proper shielding should be thoroughly considered.

The coupling coefficient [17|18] determines the amount of magnetic flux transferred from transmitter to receiver coil. It depends severely on materials and geometry of ferrite cores. In terms of soft magnetic materials [19-24], ferrite materials MnZn and NiZn are used for wireless power transfer. MnZn has high values of permeability [23] and can be used for applications where high values of the coupling coefficient are needed. However, NiZn material has lower eddy current losses what is particularly important in

\footnotetext{
${ }^{*}$ Corresponding author: Ludvik Strauch, Kolektor Group, Kolektor Sikom, Magma, Ljubljana, Stegne 29, SI-1000 Slovenia. Tel.: +386 15833 144; Fax: +386 15833 231; E-mail: ludvik.strauch@kolektor.com.
} 
high frequencies range over $100 \mathrm{kHz}$ where losses are the main factor of interest [24]. Ferrite core geometry [19]16|25] has also a huge impact on the value of the coupling coefficient, therefore optimal geometry should be chosen. Most common ferrite core geometries used in inductive system are: plates [6, 15[26], pot cores, [27|28], E-cores [8]11], and U-cores [9].

Ferrite cores have the advantage to work as magnetic shields against stray magnetic fields. Both transmitter and receiver coils produce some stray magnetic fields [29], which may interfere with the power electronics of the inductive system or other devices nearby. Proper shielding [15|30|31] to reduce stray magnetic fields should be applied according to the individual application demands. Ferrite material is often used as a magnetic shield to reduce stray magnetic fields or in combinations with other shielding methods [6]26|32].

The processing of ferrite cores for serial production is a complex process due to ferrite brittleness, cores dimensional tolerances, high prices of custom made dies, and pressing ability of machines [33]. Larger size cores are more difficult to process than smaller ones. This problem can be solved by assembling smaller size ferrite pieces into a larger one [34]. Another problem is the consumption of ferrite material which is connected with the price and weight of a final produced ferrite core [35]. The solution to this problem is numerical modeling with optimization of ferrite core geometry according to the specific application.

The aim of this paper is to present a new design of ferrite core made of ferrite bars for inductive wireless power transfer. The authors wanted to answer the question on how many ferrite bars use the least ferrite material for the same coupling coefficient $k$. This is a very important from economical reason, especially in serial production where the cost and consumption of ferrite material is the required information when designing new components. The advantages of using ferrite bars are: increase of the coupling coefficient, lower consumption of material, and reduced weight. We made seven different geometries of ferrite cores consisted of ferrite bars positioned in radial geometry on a transmitter side. With geometry optimization in 3D models using Comsol Multiphysics we determined the optimal design of ferrite bars for each of seven geometries regarding the coupling coefficient. Then, we compared two optimal design geometries of three and nine ferrite bars in terms of maximal and minimal consumption of ferrite material. Next, we parameterized different geometric parameters of ferrite bars to see impacts on the coupling coefficient and magnetic flux density on the transmitter side. Further, we analyzed the impact of angular alignment between ferrite bars on the coupling coefficient. Simulations of optimal designs were verified by measurements of the coupling coefficient and magnetic flux density on the transmitter side.

\section{Materials and methods}

Geometry made of ferrite bars on the transmitter side and ferrite plate on the receiver side is shown in Fig. 1 and in Table 1. Both transmitter and receiver coils were at the same distance of $l_{c}=2 \mathrm{~mm}$. Values of geometric parameters $a_{t}, b_{t}, c_{t}$ of ferrite bars on transmitter side, and plate with geometric parameters $a_{r}, b_{r}, c_{r}$ on receiver side are shown in Table 1. Coils are defined with inner $R_{\text {tin }}$ and outer radius $R_{\text {tout }}$ on transmitter side, and inner $R_{\text {rin }}$ and outer radius $R_{\text {rout }}$ on receiver side what is also shown in Table 1 .

Due to availability of ferrite materials NiZn material type 1C, manufacturer Kolektor with permeability $\mu_{t}=900$ and resistivity $\rho_{t}=10^{3} \Omega \cdot \mathrm{m}$ was used on transmitter side, while on receiver side there was MnZn material type $65 \mathrm{G}$, manufacturer Kolektor with $\mu_{r}=2200$ and $\rho_{r}=7 \Omega \cdot \mathrm{m}$ [36] both presented in Table 1. Prototype of $n=9$ ferrite bars made of material 1C on transmitter side is shown in Fig. 2(a), while ferrite plate made of material $65 \mathrm{G}$ on receiver side is shown in Fig. 2(b). 
Table 1

Geometric parameters and material properties

\begin{tabular}{|c|c|c|}
\hline Geometric parameters & Transmitter coil & Receiver coil \\
\hline $\begin{array}{l}\text { Number of turns } \\
\text { Coil inner radius } \\
\text { Coil outer radius }\end{array}$ & $\begin{array}{c}10 \\
R_{\mathrm{tin}}=9 \mathrm{~mm} \\
R_{\text {tout }}=15.8 \mathrm{~mm}\end{array}$ & $\begin{array}{c}20 \\
R_{\text {rin }}=9 \mathrm{~mm} \\
R_{\text {rout }}=17.5 \mathrm{~mm}\end{array}$ \\
\hline $\begin{array}{l}\text { Geometry } \\
\text { Ferrite bar (transmitter side) }\end{array}$ & \multicolumn{2}{|c|}{$\begin{array}{c}a_{\mathrm{top} 3} \times b_{t} \times c_{t} \\
16 \mathrm{~mm} \times 18 \mathrm{~mm} \times 2 \mathrm{~mm}(n=3) \\
a_{\mathrm{top} 9} \times b_{t} \times c_{t} \\
3 \mathrm{~mm} \times 18 \mathrm{~mm} \times 2 \mathrm{~mm}(n=9) \\
1 \mathrm{C}, \text { Kolektor, } \mu_{t}=900, \rho_{t}=10^{3} \Omega \cdot \mathrm{m}\end{array}$} \\
\hline Ferrite plate (receiver side) & \multicolumn{2}{|c|}{$\begin{array}{c}a_{r} \times b_{r} \times c_{r} \\
53 \mathrm{~mm} \times 53 \mathrm{~mm} \times 2.5 \mathrm{~mm} \\
65 \mathrm{G}, \text { Kolektor, } \mu_{r}=2200, \rho_{r}=7 \Omega \cdot \mathrm{m}\end{array}$} \\
\hline
\end{tabular}

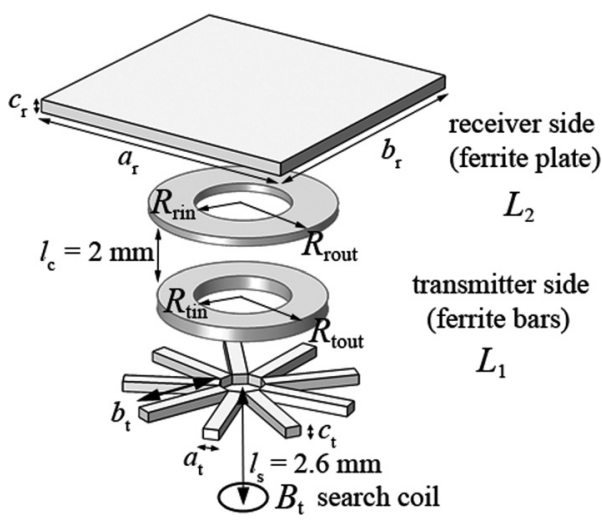

Fig. 1. Geometry made of $n=9$ ferrite bars on the transmitter side and ferrite plate on the receiver side.

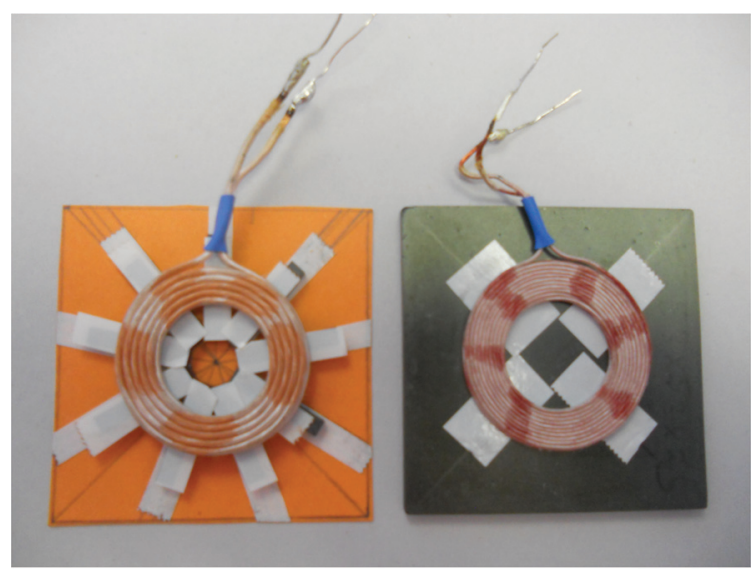

(a)

Fig. 2. Prototype of (a) $n=9$ ferrite bars with transmitter coil on the transmitter side and (b) ferrite plate with receiver coil on the receiver side.

Two parameters, the coupling coefficient $k$ and magnetic flux density on transmitter side $B_{t}$ for stray magnetic fields were used in this paper for measurements. The first parameter $k$ was used to analyze the different ferrite core geometries. The second parameter $B_{t}$ was used to analyze the optimal geometries of ferrite bars in terms of magnetic shield.

The coupling coefficient $k$ [17|18]

$$
k=\frac{M}{\sqrt{L_{1} L_{2}}}
$$

was measured by LF HP4192A impedance analyzer (Hewlett-Packard, USA) through the selfinductances $L_{1}, L_{2}$, and mutual inductance $M$. Impedance analyzer was set on frequency $150 \mathrm{kHz}$ and oscillator level of $U_{\text {osc }}=1 \mathrm{~V}$. The frequency $150 \mathrm{kHz}$ was taken as a representative value of middle frequency used in Qi standard [37], where the frequency range is defined from $100 \mathrm{kHz}$ to $205 \mathrm{kHz}$.

The mutual inductance $M$

$$
M=\frac{L_{\mathrm{a}}-L_{\mathrm{o}}}{4}
$$


was obtained with the series aiding

$$
L_{\mathrm{a}}=L_{1}+L_{2}+2 \mathrm{M}
$$

and the series opposing method

$$
L_{\mathrm{o}}=L_{1}+L_{2}-2 \mathrm{M}
$$

The coupling coefficient $k$ represents the amount of magnetic flux transferred from transmitter to receiver coil. In case of all the magnetic flux is transferred from transmitter to receiver coil $k=1$. On the other hand, if no magnetic flux is transferred, $k=0$. Therefore, the value of coupling coefficient is between the values $0<k<1$. High values of $k$ are normally required where more magnetic flux and thus energy is transferred from transmitter to receiver coil.

Magnetic flux density $B_{t}$ (amplitude) was measured by the search coil (diameter $D=6 \mathrm{~mm}$ and number of turns $N=100$, wire diameter $d=0.17 \mathrm{~mm}$, the winding wound directly on the coil former) through induced voltage $U$ (amplitude) [38]

$$
B_{t}=\frac{2 U}{N \pi^{2} D^{2} f}
$$

with oscilloscope Tektronix TDS5032B (Tektronix, USA) on a transmitter side of a ferrite core on a distance of $l_{s}=2.6 \mathrm{~mm}$, as it is shown in Fig. 1. In terms of magnetic shielding the value of $B_{t}$ is required to be as small as possible in order not to interfere with electronics positioned under the transmitter coil or functioning of electric devices nearby. The transmitter coil was powered by function generator Agilent 33220A (Agilent Technologies, USA), and power amplifier Amplifier research 700A1 (Amplifier research, USA). Sinusoidal current with amplitude of $1 \mathrm{~A}$ and frequency $150 \mathrm{kHz}$ was used. Current through the transmitter coil was measured by current probe TCP202 (Tektronix, USA).

\section{Numerical modeling}

The coupling coefficient $k$ and $B_{t}$ magnetic flux density on the transmitter side were simulated using Comsol Multiphysics 4.3 software [39] which uses the finite element method for solving partial differential equations.

In our research AC/DC Module, Magnetic Fields was used for simulation of magnetic flux density and the coupling coefficient in 3D numerical models. Coil Current Calculation, and Frequency Domain for each of the transmitter and receiver coil were used for studies of a problem in Comsol Multiphysics.

The Magnetic Fields module has equation

$$
\left(j \omega \sigma-\omega^{2} \varepsilon_{0} \varepsilon_{\mathrm{r}}\right) \mathbf{A}+\nabla \times \mathbf{H}=\mathbf{J}_{\mathrm{e}},
$$

which enables calculation of magnetic field distribution

$$
\mathbf{B}=\nabla \times \mathbf{A},
$$

where $\omega$ is the angular frequency, $\sigma$ is the electrical conductivity, $\varepsilon_{0}$ is the permittivity of vacuum, $\varepsilon_{r}$ is the relative permittivity, $\mathbf{A}$ is the magnetic vector potential, $\mathbf{H}$ is the magnetic field intensity, $\mathbf{B}$ is the magnetic flux density, and $\mathbf{J}_{\mathrm{e}}$ is the external current density. 


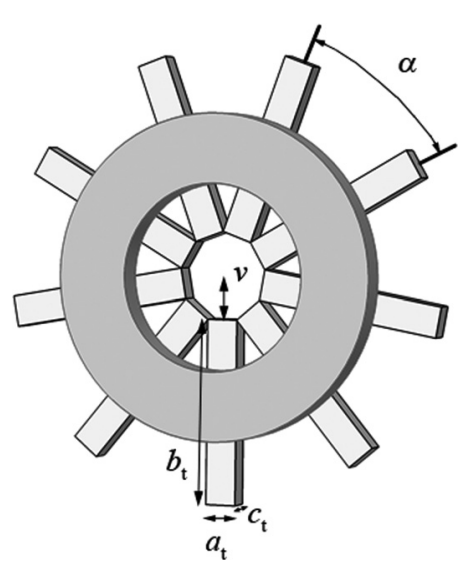

Fig. 3. Geometric parameters of ferrite bars on the transmitter side.

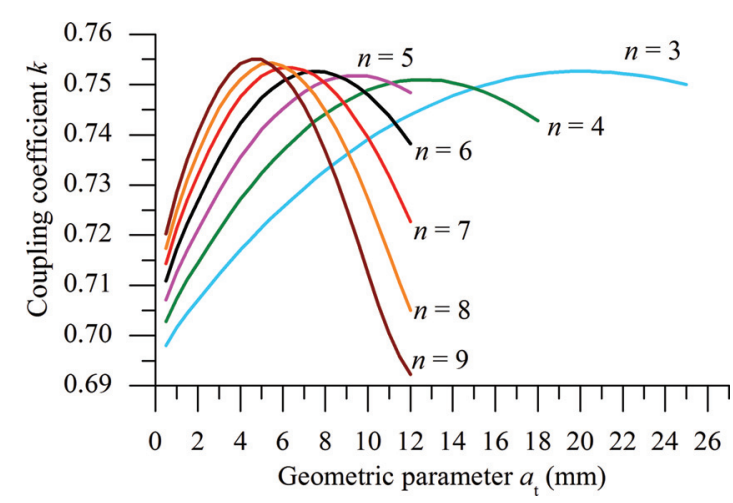

Fig. 4. Simulated values of the coupling coefficient $k$ dependence on geometric parameter $a_{t}$ for $b_{t}=18 \mathrm{~mm}, c_{t}=$ $2 \mathrm{~mm}$, and $n=3-9$.

Two models were used in Comsol Multiphysics, where in the first model we simulated self-inductance of the transmitter coil $L_{1}$, mutual inductance $M$, and magnetic flux density $B_{t}$. With the second model we simulated self-inductance of the receiver coil $L_{2}$.

The mutual inductance $M$ was determined through an equation:

$$
M=\frac{U_{2}}{j 2 \pi f I_{1}}
$$

where $U_{2}$ is induced voltage in receiver coil, $f$ frequency $(150 \mathrm{kHz})$, and $I_{1}$ is current in the transmitter coil. In the first model only the transmitter coil was excited with current amplitude of 1 A to simulate transmitter self-inductance $L_{1}$. Similarly, in the second model only the receiver coil was excited with current amplitude of $1 \mathrm{~A}$ to simulate receiver self-inductance $L_{2}$. From both models the coupling coefficient $k$ from Eq. (1) was calculated. Both transmitter and receiver coil were simulated with multi-turn coil domain for applying the number of turns. Geometry parameterizations of parameters $a_{t}, b_{t}$, and $c_{t}$ were made. Variations of parameters $a_{t}, b_{t}$, and $c_{t}$ were done with a parametric sweep in Comsol Multiphysics with a step of a $0.5 \mathrm{~mm}$. Average mesh of one geometry parameterization was made of 40000 tetrahedral elements.

The simulated values of optimal geometries of $n=3$ and $n=9$ were verified by measurements of the coupling coefficient $k$ and $B_{t}$ magnetic flux density on the transmitter side.

\section{Results and discussion}

We analyzed seven geometries with ferrite bars on transmitter side, whereas on receiver side, we used one ferrite plate for all the cases, as it is shown in Fig. 1. An example of one geometry made of $n=9$ ferrite bars with parameters $a_{t}, b_{t}, c_{t}$, and $v$ is shown in Fig. 3. Parameter $n$ represents number of ferrite bars and was parameterized in the range of $n=3-9$. When changing the parameter $n$ also the parameter $\alpha$ (the angle between ferrite bars) is changed according to the equation

$$
\alpha=\frac{2 \pi}{n} \text {. }
$$




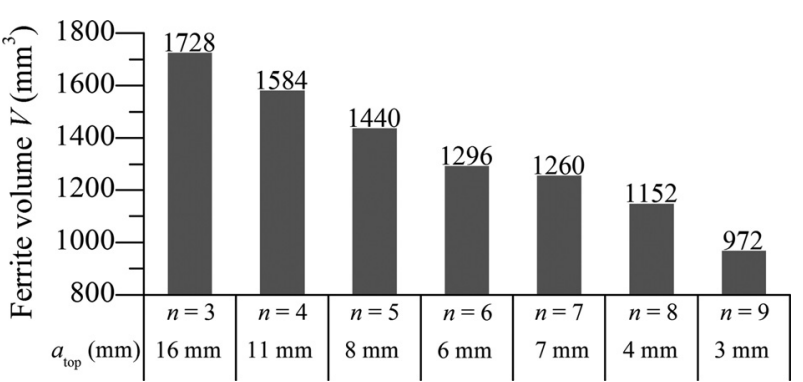

Fig. 5. Ferrite volume $V$ dependence of optimal geometric parameter $a_{\mathrm{top}}(\mathrm{mm})$ for $b_{t}=18 \mathrm{~mm}, c_{t}=2 \mathrm{~mm}, k=0.75$, and $n=3-9$.

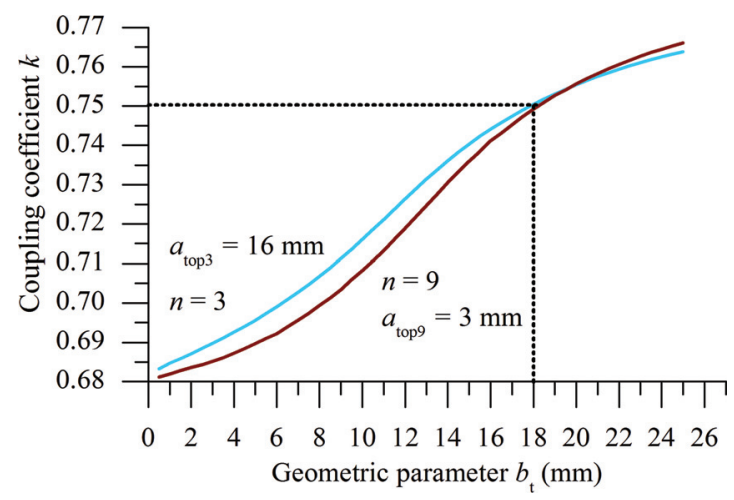

Fig. 6. Simulated values of the coupling coefficient $k$ dependence on geometric parameter $b_{t}$ for $c_{t}=2 \mathrm{~mm}$ and optimal geometries of $n=3$, and $n=9$.

During simulations in Comsol Multiphysics we optimized parameter $a_{t}$ where parameters $b_{t}=18 \mathrm{~mm}$ and $c_{t}=2 \mathrm{~mm}$ remained constant values. Parameter $v$ increased along with the increase of parameter $a_{t}$ with a step of a $0.5 \mathrm{~mm}$ according to the equation

$$
v=\frac{a_{t}}{2} \tan \left(\frac{\pi}{2}-\frac{\pi}{n}\right) .
$$

After completing the simulations, we obtained the coupling coefficient $k$ dependence on the parameter $a_{t}$ for $n=3-9$ ferrite bars geometries on transmitter side, as it is shown in Fig. 4.

Each of simulated geometry reached a maximal value of the coupling coefficient $k$ at a specific value of parameter $a_{t}$. In order to compare different geometries between each other we defined new parameter $a_{\text {top }}$. Optimal geometric parameter $a_{\text {top }}$ was defined at the value of $k=0.75$, where $k$ reached the highest value with the minimal usage of ferrite material. The value $k=0.75$ was chosen to compare different ferrite bars geometries to the same value of the coupling coefficient $k$ to have the same conditions for all the geometries. The geometries $n=3,4,5$ reached a maximum value of the coupling coefficient at $k=$ 0.75 . In comparison, geometries $n=6,7,8$, and 9 reached a maximum value of the coupling coefficient between $k=0.75$ and $k=0.76$. With value $k=0.75$ all the described geometries of ferrite bars were covered. The value of $k=0.75$ is associated with only this specific geometry of coils with ferrite cores and the distance between transmitter and receiver coil of $l_{\mathrm{c}}=2 \mathrm{~mm}$.

Ferrite bars geometries were compared according to the value of ferrite volume on transmitter side as

$$
V=n a_{\mathrm{top}} b_{t} c_{t} .
$$

Maximum ferrite volume $V=1728 \mathrm{~mm}^{3}$ used geometry with $n=3$ and $a_{\mathrm{top} 3}=16 \mathrm{~mm}$, whereas the minimum ferrite volume $V=972 \mathrm{~mm}^{3}$ used geometry with $n=9$ and $a_{\text {top } 9}=3 \mathrm{~mm}$, as it is shown in Fig. 5.

Both optimal geometries $n=3$ and $n=9$ were taken for further simulations as they present two extreme values obtained by ferrite volume calculation. In case of increasing number of ferrite bars by parameter $n$ over $n=9$ ferrite bars the ferrite volume will still decrease. However, ferrite bars will become more brittle, leading to the problem of production of such ferrite bars. Other parameters $b_{t}$ and $c_{t}$ had an effect on consumption of ferrite material according to Eq. (11).

Parameter $b_{t}$ was simulated for optimal values of parameters $a_{\mathrm{top} 3}=16 \mathrm{~mm}$ for $n=3$ and $a_{\mathrm{top} 9}=$ $3 \mathrm{~mm}$ for $n=9$, where $c_{t}=2 \mathrm{~mm}$ for both geometries, as it is shown in Fig. 5. The difference between 


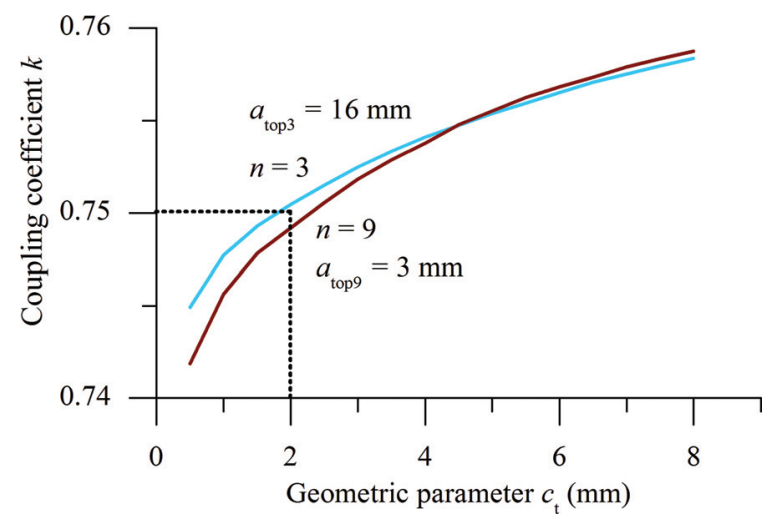

Fig. 7. Simulated values of the coupling coefficient $k$ dependence on geometric parameter $c_{t}$ for $b_{t}=18 \mathrm{~mm}$ and optimal geometries of $n=3$, and $n=9$.

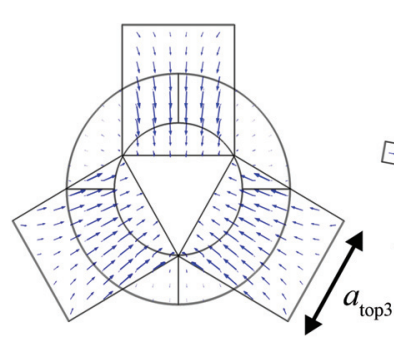

(a)

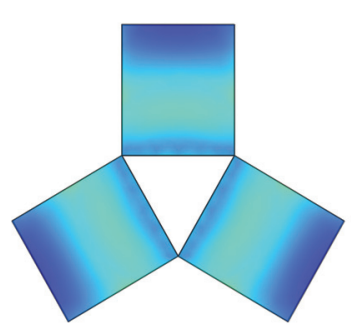

(c)

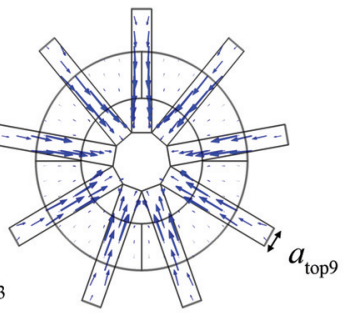

(b)

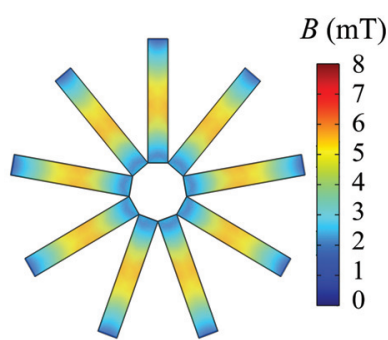

(d)
Fig. 8. Optimal geometries of (a), (c) for $n=3$ and (b), (d) for $n=9$ ferrite bars with different magnetic flux density distributions, for $k=0.75$.

both geometries regarding $k$ is less than $1 \%$, as it is shown in Fig. 6 . The value of the parameter $b_{t}=$ $18 \mathrm{~mm}$ was taken for further research as the coupling coefficient reached value $k=0.75$. There is a practical reason why not to increase the parameter $b_{t}$, which is connected with a free space limitation of coil housing. If parameter $b_{t}$ is increased over the value of transmitter coil radius $R_{\text {tout }}$ too much, then the coil will not fit into the housing. Therefore, some compromise has to be considered when increasing the value of parameter $b_{t}$.

Next, we simulated variation of parameter $c_{t}$ for optimal geometries $a_{\mathrm{top} 3}=16 \mathrm{~mm}$ for $n=3$ and $a_{\mathrm{top} 9}=3 \mathrm{~mm}$ for $n=9$, where $b_{t}=18 \mathrm{~mm}$ for both geometries, as it is shown in Fig. 7. Similarly, as discussed before by variation of parameter $b_{t}$, parameter $c_{t}$ has even minor difference regarding the coupling coefficient well below $0.1 \%$. The value of parameter $c_{t}=2 \mathrm{~mm}$ was taken for further research as $k=0.75$. Variations of parameter $c_{t}$ were done, because in Qi standard there are different minimal ferrite thicknesses, for example $0.5 \mathrm{~mm}, 3.1 \mathrm{~mm}$, and $5 \mathrm{~mm}$ specified according to the different sizes and geometries of coils [37]. The parameter $c_{t}$ almost does not influence the coupling coefficient $k$, where increase from $c_{t}=2 \mathrm{~mm}$ to $c_{t}=8 \mathrm{~mm}$ leads to increase only by 0.1 from $k=0.75$ to $k=0.76$, as it is shown in Fig. 7.

Parameters $b_{t}$ and $c_{t}$ had a minor effect on the coupling coefficient $k$ in comparison with parameter $a_{t}$. As it can be seen in Fig. 5, optimal geometry with $n=9$ bars uses two times less ferrite material than $n=3$ bars. The difference in ferrite volume for $n=3$ and $n=9$ bars geometries can be explained in Fig. 8.

Magnetic flux density flows throughout the bars and it goes to the center of transmitter coil, as it is shown in Figs 8(a) and (b). Magnetic flux is then transferred from transmitter to receiver coil. If we want to have a value of the coupling coefficient $k=0.75$ for only $n=3$ ferrite bars in Fig. 8(a) parameter $a_{\mathrm{top} 3}$ has to be increased, while the $a_{\mathrm{top} 9}$ in Fig. $8(\mathrm{~b})$ is constant. Therefore, the same value of magnetic flux will be transferred from transmitter to receiver coil for the different consumption of ferrite material. 
Table 2

Simulated and measured values of the coupling coefficient $k$ and magnetic flux density on the transmitter side $B_{t}$ for: no ferrite, $n=3$, and $n=9$ optimal ferrite bars geometries

\begin{tabular}{ccccc}
\hline Geometry on transmitter side & $k$ (simulated) & $k$ (measured) & $B_{t}(\mu \mathrm{T})$ (simulated) & $B_{t}(\mu \mathrm{T})$ (measured) \\
\hline No ferrite & 0.68 & 0.61 & 458 & 525 \\
$n=3$ & 0.75 & 0.69 & 234 & 224 \\
$n=9$ & 0.75 & 0.69 & 179 & 167 \\
\hline
\end{tabular}

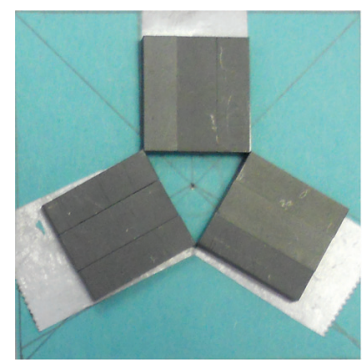

(a)

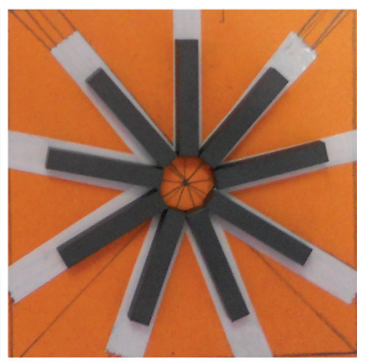

(b)

Fig. 9. Prototypes of optimal geometries: (a) $n=3$ ferrite bars, (b) $n=9$ ferrite bars.

Simulated values of optimal geometries $a_{\mathrm{top} 3}=16 \mathrm{~mm}$ for $n=3$ and $a_{\mathrm{top} 9}=3 \mathrm{~mm}$ for $n=9$, where $b_{t}=18 \mathrm{~mm}, c_{t}=2 \mathrm{~mm}$ for both geometries were verified by measurements in Table 2 . Optimal geometries $n=3$ and $n=9$ have higher values of $k=0.75$ in comparison with no ferrite geometry on transmitter side, where $k=0.68$ for simulated values. More detailed information about types of ferrite materials and geometries are presented in Table 1.

In terms of magnetic shielding, magnetic flux density on the transmitter side of a coil $B_{t}$ was measured and simulated. Prototypes of optimal geometries $n=3$ and $n=9$ are shown in Fig. 9. Optimal geometry $n=9$ has three times the lower value of $B_{t}=167 \mu \mathrm{T}$ in comparison with no ferrite geometry $B_{t}=$ $525 \mu \mathrm{T}$ for measured values, as presented in Table 2. According to the Table 2, optimal geometry $n=9$ is better magnetic shield than $n=3$ geometry, where no ferrite geometry has the highest value of $B_{t}$.

There are the differences between simulated and measured values of the coupling coefficient $k$ and magnetic flux density on the transmitter side $B_{t}$ in Table 2. The reasons for the difference of $k$ are additional wires of transmitter and receiver coils in prototypes, and wires connecting those coils to the impedance analyzer LF HP4192A. In contrast, the simulated models do not take into account additional wires in Comsol Multiphysics. The reason for the difference between simulated and measured values $B_{t}$ is that the magnetic field was not measured directly. Parameter $B_{t}$ was measured through induced voltage according to the Eq. (5). There exist many definitions of which value to take for the diameter of a search coil [38]. In this paper, mean value of the diameter of a search coil was taken for the calculation of a magnetic field $B_{t}$. Therefore, the selection alone of a diameter of search coil can seriously affect the final calculated value of magnetic field, which leads to the difference between simulated and measured values of parameter $B_{t}$.

Magnetic flux density distribution of optimal geometries for $n=3$ and $n=9$ are presented in Fig. 8(c) and in Fig. 8(d). In both geometries higher values of magnetic flux density $B$ are in the position of transmitter coils, where the source of magnetic flux is stationed. Optimal geometry with $n=3$ in Fig. 8(c) has values of $B$ in the range of $3 \mathrm{mT}$, where for $n=9$ are in the range of $6 \mathrm{mT}$ in Fig. 8(d). Both 
values of $B$ are too low that ferrite can go into saturation, as for that kind of ferrite material, the value of magnetic flux density saturation is specified in the range of $350 \mathrm{mT}$.

Until now ferrite bars geometry was only on transmitter side, whereas on receiver side there was a ferrite plate. In case of mobile devices, it would be interesting to see what happens if the ferrite bars geometry is also on the receiver side. Therefore, we simulated ferrite bars on the receiver side for the different angle $\beta$, as it is shown in Fig. 10. In that case $\beta$ means the angle between ferrite bars on transmitter and receiver side. Effect of angular alignment has a lower effect for the optimal geometry of $n=9$ ferrite bars, which has a difference in the coupling coefficient $\Delta k=0.13 \%$, what is ten times lower than the optimal geometry of $n=3$ ferrite bars.

\section{Conclusions}

In summary, new design of ferrite core made of ferrite bars for inductive wireless power transfer is presented in this paper. Seven different geometries of ferrite bars in radial geometry were investigated. Parameterizations of geometric parameters were done with 3D models in Comsol Multiphysics, which uses the finite element method. Two optimal geometries with three and nine ferrite bars were thoroughly analyzed. Simulations for both geometries and also with no ferrite geometry on transmitter side were verified by measurements.

Optimal geometry with nine ferrite bars in radial geometry is proposed as it has a high coupling coefficient, low consumption of ferrite material, and reduced weight. In terms of magnetic shield nine ferrite bars in radial geometry is three times better magnetic shield than no ferrite geometry for measuring values. Values of magnetic flux density in ferrite bars are low enough that the ferrite core does not saturate.

The angular alignment of ferrite bars in radial geometry on the transmitter and the receiver side was negligible and had difference in the coupling coefficient $1.35 \%$ for three ferrite bars geometry and $0.13 \%$ for nine ferrite bars for simulated values.

\section{References}

[1] M. Budhia, G.A. Covic and J.T. Boys, Design and optimization of circular magnetic structures for lumped inductive power transfer systems, IEEE Transactions on Power Electronics 26(11) (2011), 3096-3108, doi 10.1109/TPEL.2011. 2143730.

[2] G.A. Covic and J.T. Boys, Inductive power transfer, Proceedings of the IEEE 101(6) (2013), 1276-1289, doi 10.1109/ JPROC.2013.2244536.

[3] C. Hu, Y. Sun, Z. Wang, C. Tang and Q. Xiong, Design of magnetic coupler for EVs' wireless charging, International Journal of Applied Electromagnetics and Mechanics 43(3) (2013), 195-205, doi 10.3233/JAE-131693.

[4] M. Ibrahim, L. Bernard, L. Pichon and A. Razek, Electromagnetic model of EV wireless charging systems in view of energy transfer and radiated field control, International Journal of Applied Electromagnetics and Mechanics 46(2) (2014), 355-360, doi 10.3233/JAE-141945.

[5] C. Hu, Y. Sun, X. Lv, Z. Wang and Q. Xiong, Magnetic coupler design procedure for IPT system and its application to EVs' wireless charging, International Journal of Applied Electromagnetics and Mechanics 47(3) (2015), 861-873, doi: 10.3233/JAE-140146.

[6] S. Kim, H.H. Park, J. Kim, J. Kim and S. Ahn, Design and analysis of a resonant reactive shield for a wireless power electric vehicle, IEEE Transactions on Microwave Theory and Techniques 62(4) (2014), 1057-1066, doi 10.1109/TMTT.2014.2305404.

[7] H. Hasegawa, T. Kashiwagi, Y. Sakamoto and T. Sasakawa, Study of magnet rotor type non-contact transformer, International Journal of Applied Electromagnetics and Mechanics 33(1) (2010), 135-144, doi 10.3233/JAE-2010-1106.

[8] J.T. Boys, G.A. Covic and A.W. Green, Stability and control of inductively coupled power transfer systems, IEE Proceedings - Electric Power Applications 147(1) (2000), 37-43, doi 10.1049/ip-epa:20000017. 
[9] G.A.J. Elliot, G.A. Covic, D. Kacprzak and J.T. Boys, A new concept: Asymmetrical pick-ups for inductively coupled power transfer monorail systems, IEEE Transactions on Magnetics 42(10) (2006), 3389-3391, doi 10.1109/TMAG. 2006. 879619.

[10] C.B. Park, H.W. Lee and B.S. Lee, A study on the design parameters of inductive power transformers, International Journal of Applied Electromagnetics and Mechanics 39(1) (2012), 809-815, doi 10.3233/JAE-2012-1546.

[11] C.G. Kim, D.H. Seo, J.S. You, J.H. Park and B.H. Cho, Design of a contactless battery charger for cellular phone, IEEE Transactions on Industrial Electronics 48(6) (2001), 1238-1247, doi 10.1109/41.969404.

[12] E. Waffenschmidt and T. Staring, Limitation of inductive power transfer for consumer applications, Proceedings of the 13th European Conference on Power Electronics and Applications (2009), 1-10.

[13] S.Y.R. Hui and W.W.C. Ho, A new generation of universal contactless battery charging platform for portable consumer electronic equipment, IEEE Transactions on Power Electronics 20(3) (2005), 620-627, doi 10.1109/TPEL.2005.846550.

[14] S.Y.R. Hui, Planar wireless charging technology for portable electronic products and Qi, Proceedings of the IEEE 101(6) (2013), 1290-1301, doi 10.1109/JPROC.2013.2246531.

[15] Y.P. Su, X. Liu and S.Y.R. Hui, Extended theory on the inductance calculation of planar spiral windings including the effect of double-layer electromagnetic shield, IEEE Transactions on Power Electronics 23(4) (2008), 2052-2061, doi 10.1109/TPEL.2008.924824.

[16] J. Acero, C. Carretero, I. Lope, R. Alonso, O. Lucia and J.M. Burdio, Analysis of the mutual inductance of planar-lumped inductive power transfer systems, IEEE Transactions on Industrial Electronics 60(1) (2013), 410-420, doi 10.1109/TIE. 2011.2164772.

[17] S. Djuric, G. Stojanovic, M. Damnjanovic, M. Radovanovic and E. Laboure, Design, modeling and analysis of a compact planar transformer, IEEE Transactions on Magnetics 48(11) (2012), 4135-4138, doi 10.1109/TMAG.2012.2202642.

[18] S. Djuric, G. Stojanovic, M. Damnjanovic and E. Laboure, Analysis of the coupling effect in different meander-type winding planar transformers, IEEE Transactions on Magnetics 49(7) (2013), 3993-3996, doi 10.1109/TMAG.2013. 2243417.

[19] A.M. Leary, P.R. Ohodnicki and M.E. McHenry, Soft magnetic materials in high-frequency, high-power conversion applications, JOM 64(7) (2012), 772-781, doi 10.1007/s11837-012-0350-0.

[20] M.E. McHenry and D.E. Laughlin, Magnetic moment and magnetization, in: Characterization of Materials, John Wiley and Sons, New York, 2012, pp. 1-25.

[21] M.E. McHenry, M.A. Willard and D.E. Laughlin, Amorphous and nanocrystalline materials for applications as soft magnets, Progress in Materials Science 44(4) (1999), 291-433, doi 10.1016/S0079-6425(99)00002-X.

[22] E.C. Snelling, Soft Ferrites: Properties and Applications, First edition, Iliffe Books, London, England, 1969, pp. 38-171.

[23] A. Goldman, Modern Ferrite Technology, Second edition, Springer, New York, USA, 2006, pp. 35-50.

[24] M.K. Kazimierczuk, High-frequency magnetic components, first edition, John Wiley and Sons, Chichester, United Kingdom, 2009, pp. 49-89.

[25] S.M. Djuric and G.M. Stojanovic, A compact planar transformer with an improved winding configuration, IEEE Transactions on Magnetics 50(11) (2014), 1-4, doi 10.1109/TMAG.2014.2321630.

[26] S.C. Tang, S.Y.R. Hui and H.S.H. Chung, Evaluation of the shielding effects on printed-circuit-board transformers using ferrite plates and copper sheets, IEEE Transactions on Power Electronics 17(6) (2002), 1080-1088, doi 10.1109/TPEL. 2002.805585.

[27] D. Kurschner, C. Rathge and U. Jumar, Design methodology for high efficient inductive power transfer systems with high coil positioning flexibility, IEEE Transactions on Industrial Electronics 60(1) (2013), 372-381, doi 10.1109/TIE. 2011. 2181134

[28] A.J. Moradewicz and M.P. Kazmierkowski, Contactless energy transfer system with FPGA-controlled resonant converter, IEEE Transactions on Industrial Electronics 57(9) (2010), 3181-3190, doi 10.1109/TIE.2010.2051395.

[29] P. Sergeant, L. Dupre and J. Melkebeek, Active and passive magnetic shielding for stray field reduction of an induction heater with axial flux, IEE Proceedings - Electric Power Applications 152(5) (2005), 1359-1364, doi 10.1049/ipepa:20050005.

[30] J. Kim, J. Kim, S. Kong, H. Kim, I.S. Suh, N.P. Suh, D.H. Cho, J. Kim and S. Ahn, Coil design and shielding methods for a magnetic resonant wireless power transfer system, Proceedings of the IEEE 101(6) (2013), 1332-1342, doi 10.1109/JPROC.2013.2247551.

[31] Y.L. Bihan, Analytical model of a shielded U-core sensor, International Journal of Applied Electromagnetics and Mechanics 19(1) (2004), 99-102.

[32] T. Saito, Open-type magnetic shielding method, International Journal of Applied Electromagnetics and Mechanics 33(3) (2010), 891-899, doi 10.3233/JAE-2010-1199.

[33] R. Severns, E. Yeow, G. Woody, J. Hall and J. Hayes, An ultra-compact transformer for a $100 \mathrm{~W}$ to $120 \mathrm{~kW}$ inductive coupler for electric vehicle battery charging, Proceedings of the 11th Applied Power Electronics Conference and Exposition 1 (1996), 32-38, doi 10.1109/APEC.1996.500418.

[34] Y. Matsuo, O.M. Kondoh and F. Nakao, Controlling new die mechanisms for magnetic characteristics of super-large 
ferrite cores, IEEE Transactions on Magnetics 36(5) (2000), 3411-3414, doi 10.1109/20.908843.

[35] F. Nakao, Y. Matsuo, M. Kitaoka and H. Sakamoto, Ferrite core couplers for inductive chargers, Proceedings of the Power Conversion Conference 2 (2002), 850-854, doi 10.1109/PCC.2002.997631.

[36] Kolektor 1C, 65G materials. [Online]. Available: http://www.kolektor.com/resources/files/doc/Products_overview_PDF_ .pdf, (2015, May 14).

[37] System Description Wireless Power Transfer, Volume I: Low Power Part 1: Interface Definition Version 1.1.2, June 2013, pp. 1, 12, 42, 25.

[38] S. Tumanski, Induction coil sensors-a review, Measurement Science and Technology 18 (2007), R31-R46, doi 10.1088/ 0957-0233/18/3/R01.

[39] Comsol Multiphysics. [Online]. Available: http://www.comsol.com/products/4.3/, (2015, May 15). 